ACCEPTED MANUSCRIPT

\title{
Basic restrictions for theories of ion-induced track formation: ignored relationships between experimental data
}

To cite this article before publication: Gyorgy Szenes et al 2019 Phys. Scr. in press https://doi.org/10.1088/1402-4896/ab300c

\section{Manuscript version: Accepted Manuscript}

Accepted Manuscript is "the version of the article accepted for publication including all changes made as a result of the peer review process, and which may also include the addition to the article by IOP Publishing of a header, an article ID, a cover sheet and/or an 'Accepted

Manuscript' watermark, but excluding any other editing, typesetting or other changes made by IOP Publishing and/or its licensors"

This Accepted Manuscript is @ 2019 IOP Publishing Ltd.

During the embargo period (the 12 month period from the publication of the Version of Record of this article), the Accepted Manuscript is fully protected by copyright and cannot be reused or reposted elsewhere.

As the Version of Record of this article is going to be / has been published on a subscription basis, this Accepted Manuscript is available for reuse under a CC BY-NC-ND 3.0 licence after the 12 month embargo period.

After the embargo period, everyone is permitted to use copy and redistribute this article for non-commercial purposes only, provided that they adhere to all the terms of the licence https://creativecommons.org/licences/by-nc-nd/3.0

Although reasonable endeavours have been taken to obtain all necessary permissions from third parties to include their copyrighted content within this article, their full citation and copyright line may not be present in this Accepted Manuscript version. Before using any content from this article, please refer to the Version of Record on IOPscience once published for full citation and copyright details, as permissions will likely be required. All third party content is fully copyright protected, unless specifically stated otherwise in the figure caption in the Version of Record.

View the article online for updates and enhancements. 
Basic restrictions for theories of ion-induced track formation: ignored relationships between experimental data

${ }^{1}$ Department of Materials Physics, Eötvös University, P.O. Box 32, H-1518 Budapest,

Corresponding author

\section{Hungary}

${ }^{2}$ Institute for Technical Physics and Materials Science, Centre for Energy

Research, HAS, H-1525 Budapest, P.O. Box 49, Hungary

Gyorgy Szenes.

Department of Materials Physics,

Eötvös University,

1518 Budapest, P.O. Box 32, Hungary

phone: 36-1-372-2821

fax: 36-1-372-2811

szenesgyorgy@caesar.elte.hu 


\section{Introduction}

Ion irradiation by energetic ions may induce severe changes in insulators and intensive research activity is going on continuously for several years in various fields: track formation, swelling, electronic mixing and sputtering, formation of hillocks, anisotropic growth, radiotherapy [1]. From a theoretical point of view these effects are the results of very complex processes, as the ions may have velocities up to $10 \%$ of the velocity of light, when they lose a high number of electrons. As a result, the highly charged, fast projectile induces a very strong electromagnetic field leading to high ionization of the lattice atoms, high number of excited energetic electrons, formation of high electric field and forces, formation of deformation waves and various types of defects. A high transient temperature field is also formed during the relaxation of the system and this may lead to various processes in the solids. Besides the theoretical interests the researchers are also inspired by practical problems as well, as a growing number of radiation sources are applied for scientific, technical and medical purposes.

When an energetic ion hits a solid target it interacts with it by the elastic mechanism characterized by the nuclear stopping power $S_{n}$ and the inelastic mechanism characterized by the electronic stopping power $S_{\mathrm{e}}$. The induced damage is the result of both effects. Typically, tracks are formed in crystalline insulators where often a cylindrical amorphous phase is formed along the trajectory of a high energy projectile. The track radius $R_{e}$ depends on $S_{e}$ when $S_{e}>>S_{n}$ as the simultaneous effect of the two mechanisms is not significant for such irradiations.

Numerous theoretical approaches have been proposed up to now for explaining the track formation [2]. Though they seem to be reasonable we cannot be sure that the correct one is among them. Just the relatively high number of such models is the indication of the uncertainty concerning their validity. It is timely to cite Einstein, who wrote ,the most beautiful logical theory means nothing in natural science without comparison with the experience"[3]. Here he speaks about experience that means an investigation from many aspects. We refer to this idea because it is frequently ignored and if a calculation reproduces some experimental result in a solid it is already considered as a sufficient evidence for the validity of the applied model. 
The basic idea of this paper: any theory must be checked by various types of experiments involving many solids. Any limitation of the experimental basis reduces the value of the comparison with the experience. In the field of irradiation induced effects, the most accurate data are available for experiments of track formation [2], therefore, our analysis is based on such type of measurements. We show the results of some types of experiments which have been ignored in checking theoretical predictions but we consider them highly important. Our opinion is that the information available from experiments have not been fully utilized for verification of the various track models/ Here we demonstrate that the appropriate analysis of the experimental data is suitable for providing basically new information for theories.

In this paper, we intentionally do not apply any model and try to avoid even mentioning them. We expect new additional information from experiments performed on different materials comparing their response. When the comparison is made under appropriate conditions quantitative relationship is found between track radii measured in different track forming insulators induced in independent experiments by different ions of different energies having different values of $S_{e}$. The mathematical consequences of this relationship between nominally independent track radii are discussed providing information on the mechanism of track formation, the control parameter and the relation between the temperatures induced in different solids.

We start with experiments where tracks are induced simultaneously both by $S_{e}$ and $S_{n}$ and show that the deduced information puts several formal restrictions on the theories. When it was necessary, the available data were completed with some new ones. Then the analysis is extended to the collective features of track formation that also leads to important additional information.

\section{Experimental}

In the last three decades many experiments have been performed for measuring ion-induced tracks in various solids. Unfortunately only few of them are systematic ones and usually the ranges of 
the experimental variables are rather limited. Most of the track data were obtained with the application of high energy ions with $\mathrm{E}>1 \mathrm{MeV} /$ nucleon, when $\mathrm{S}_{\mathrm{e}}>>\mathrm{S}_{\mathrm{n}}$. However, at lower ion energies this is not the case and it is still an open question how to combine correctly the two contributions of the energy deposition. This is an important problem both from theoretical and experimental aspects. Therefore, we completed the existing data with new experimental results. $\mathrm{Y}_{3} \mathrm{Fe}_{5} \mathrm{O}_{12}$ (YIG) was chosen for the experiments as the response to irradiations by energetic heavy ions of this solid has been investigated most thoroughly.

In our experiments an YIG layer was grown on $\mathrm{Gd}_{3} \mathrm{Ga}_{5} \mathrm{O}_{12}$ (GGG) substrate by liquid phase epitaxy. The samples prethinned for transmission electron microscopy (TEM) in the Institute for Technical Physics and Materials Science, Hungarian Academy of Sciences were irradiated at room temperature by $\mathrm{C} 60$ ions of 3.5, 5 and $7 \mathrm{MeV}$ energy at the TANDEM facility in ETH Zürich. The beam parameters are given in Table 1 and the SRIM2013 code is applied for the calculation of $S_{e}$ and $S_{n}$ [4].

\begin{tabular}{|c|c|c|c|c|c|}
\hline Projectile & $\begin{array}{c}\text { Energy } \\
\mathrm{MeV}\end{array}$ & $\begin{array}{c}\mathrm{S}_{\mathrm{e}} \\
\mathrm{keV} / \mathrm{nm}\end{array}$ & $\begin{array}{c}\mathrm{S}_{\mathrm{n}} \\
\mathrm{keV} / \mathrm{nm}\end{array}$ & $\mathrm{S}_{\mathrm{n}} / \mathrm{S}_{\mathrm{e}}$ & $\begin{array}{c}\mathrm{R}_{\mathrm{e}} \\
\mathrm{nm}\end{array}$ \\
\hline $\mathrm{C} 60$ & 3.5 & 21 & 6.1 & 0.29 & 7.1 \\
\hline C60 & 5 & 25.1 & 5.1 & 0.20 & 7.1 \\
\hline C60 & 7 & 29.4 & 4.2 & 0.14 & 7.4 \\
\hline
\end{tabular}

Table 1. Irradiation parameters of prethinned TEM samples;

$\mathrm{R}_{\mathrm{e}}$ - track radius.

The fluences were in the range of $(2-4) \times 10^{10}$ ions $/ \mathrm{cm}^{2}$ to avoid track overlapping. The ion energies were chosen to have relatively high values for the ratio $S_{\mathrm{n}} / \mathrm{S}_{\mathrm{e}}$.

A Philips CM20 electron microscope was used in the studies of the irradiated YIG crystals. Although this is a conventional TEM (with a point resolution of only $0.28 \mathrm{~nm}$ at an accelerating voltage 
of $200 \mathrm{kV}$ ), due to the large lattice parameter of the investigated crystals it was completely suitable to use it in high resolution mode. In some cases high resolution electron microscopy (HREM) was also performed by means of a dedicated HRTEM made by JEOL (type 3011). This latter was used at $300 \mathrm{kV}$ accelerating voltage with a point resolution of $0.17 \mathrm{~nm}$.

Due to the preferential thinning no further preparation steps was necessary. Exact (111) orientation of the cubic YIG crystal was easily achieved by the diffraction available in the TEM. The three [110] type lattice plane series of the YIG phase $(\mathrm{d}=0.875 \mathrm{~nm})$ were easily resolved with the CM20 microscope and could be used as an internal standard for the size measurement of the tracks. The boundary between amorphous tracks and the crystal was found sharp therefore their size could be determined quite accurately.

\section{Results}

In Fig. 1 a TEM picture of a sample irradiated by $5 \mathrm{MeV}$ C60 ions is shown with uniform circular tracks. The parallel crystalline planes are clearly seen on the magnified insert and they are used for internal calibration that is highly advantageous for the accuracy of the measurements. The track diameters were determined as an average of about 50 measurements.

HREM measurements were also performed. A picture made using a JEOL 3011 electron microscope is shown in Fig.2. 


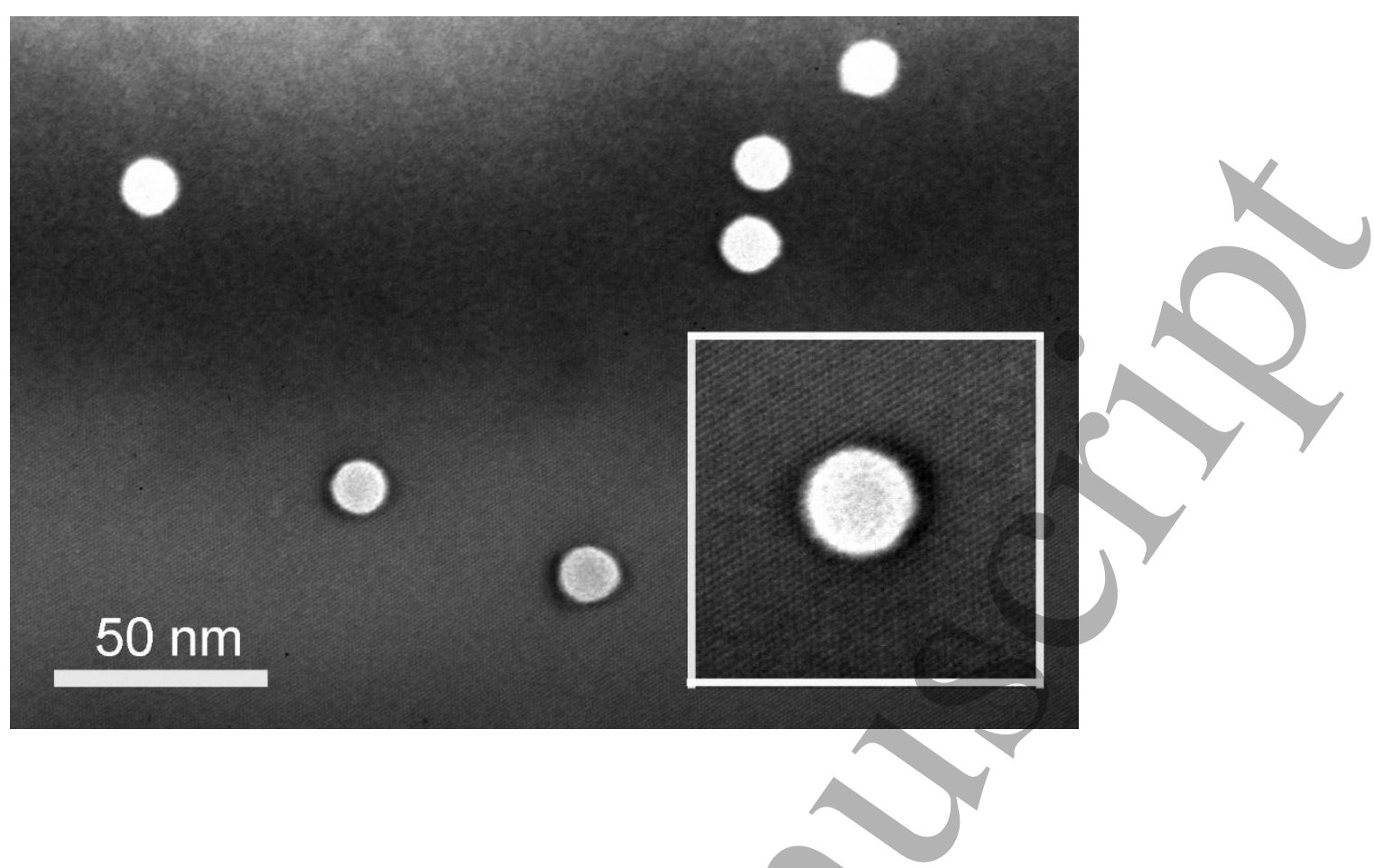

Fig.1 Bright field TEM picture of a YIG sample irradiated by $\mathrm{C} 60$ ions of $5 \mathrm{MeV}$ energy. Insert shows magnified image of the YIG crystal in $\{111\}$ orientation with a circular amorphous track.

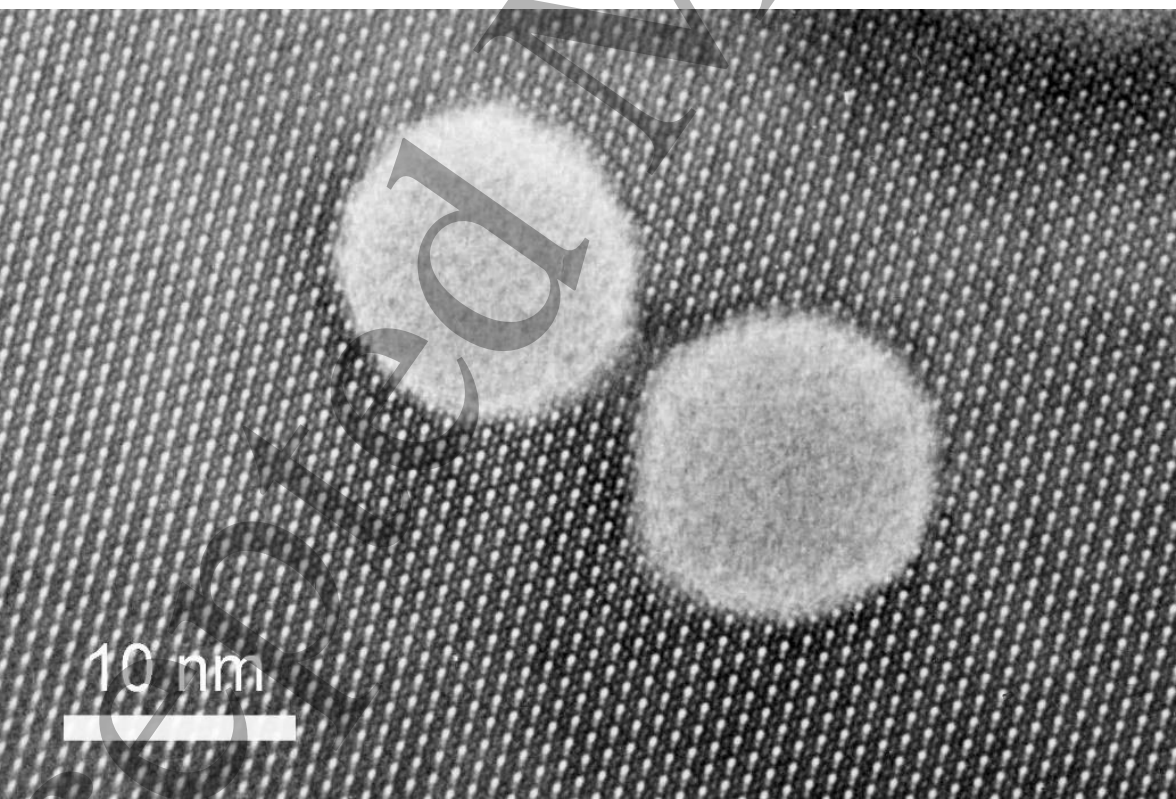

Fig.2 HREM picture of two closely positioned track in a YIG sample irradiated by C60 ions of $3.5 \mathrm{MeV}$

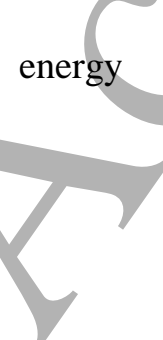


In this figure, the directions and distances of the crystal planes are well defined. The figure nicely demonstrates the amorphous structure of the tracks and the crystalline -amorphous boundary is also clearly seen. There is no marked deformation contrast around the tracks.

Compared to previous results, the track radii are considerably larger than those which have been induced by swift heavy ions at similar values of $S_{e}$ when $S_{e}>>S_{n}$. This is in agreement with the expected elastic contribution to the energy deposition. The deviations are especially emphasized when the results are compared with those of Jensen et al. [5] (see Fig.3) who have published a systematic study of tracks in YIG samples irradiated by Cn cluster ions in a broad range of $\mathrm{S}_{\mathrm{e}}$. Regretfully, similar studies on other materials are missing, at present.

\section{Discussion}

\subsection{Combination of the electronic and nuclear stopping in track formation}

In the case of dominant electronic excitation, track formation starts above a threshold value $S_{\text {et }}$ which varies with the individual materials parameters (MPs). Usually, this is a simple smooth curve and track radii $\mathrm{R}_{\mathrm{e}}$ vary monotonously with $\mathrm{S}_{\mathrm{e}}$. One of the purposes of this paper is to get information how $\mathrm{S}_{\mathrm{e}}$ and $\mathrm{S}_{\mathrm{n}}$ are combined when they act simultaneously. This is quite different from the situation when a sample is preirradiated by low energy ions and then it is irradiated by high energy projectiles in a second step.

First, we studied the results of previous experiments in various insulators for $S_{\mathrm{e}}$ values well above $\mathrm{S}_{\mathrm{et}}$ and $\mathrm{S}_{\mathrm{e}}>>\mathrm{S}_{\mathrm{n}}$ and such experimental results are shown in Ref.[6]. These conditions were satisfied by monoatomic projectiles and energetic cluster ions as well. Compared to the intensity of the research for several decades in this field, the number of suitable systematic studies is relatively low. We selected tracks induced by ions in the range of about $\mathrm{E}<2 \mathrm{MeV} /$ nucleon. The importance of this condition will be discussed later. In Fig.2 in Ref.[6], the $\mathrm{R}_{\mathrm{e}}{ }^{2}-\mathrm{S}_{\mathrm{e}}$ track evolution curves can be described in a good 


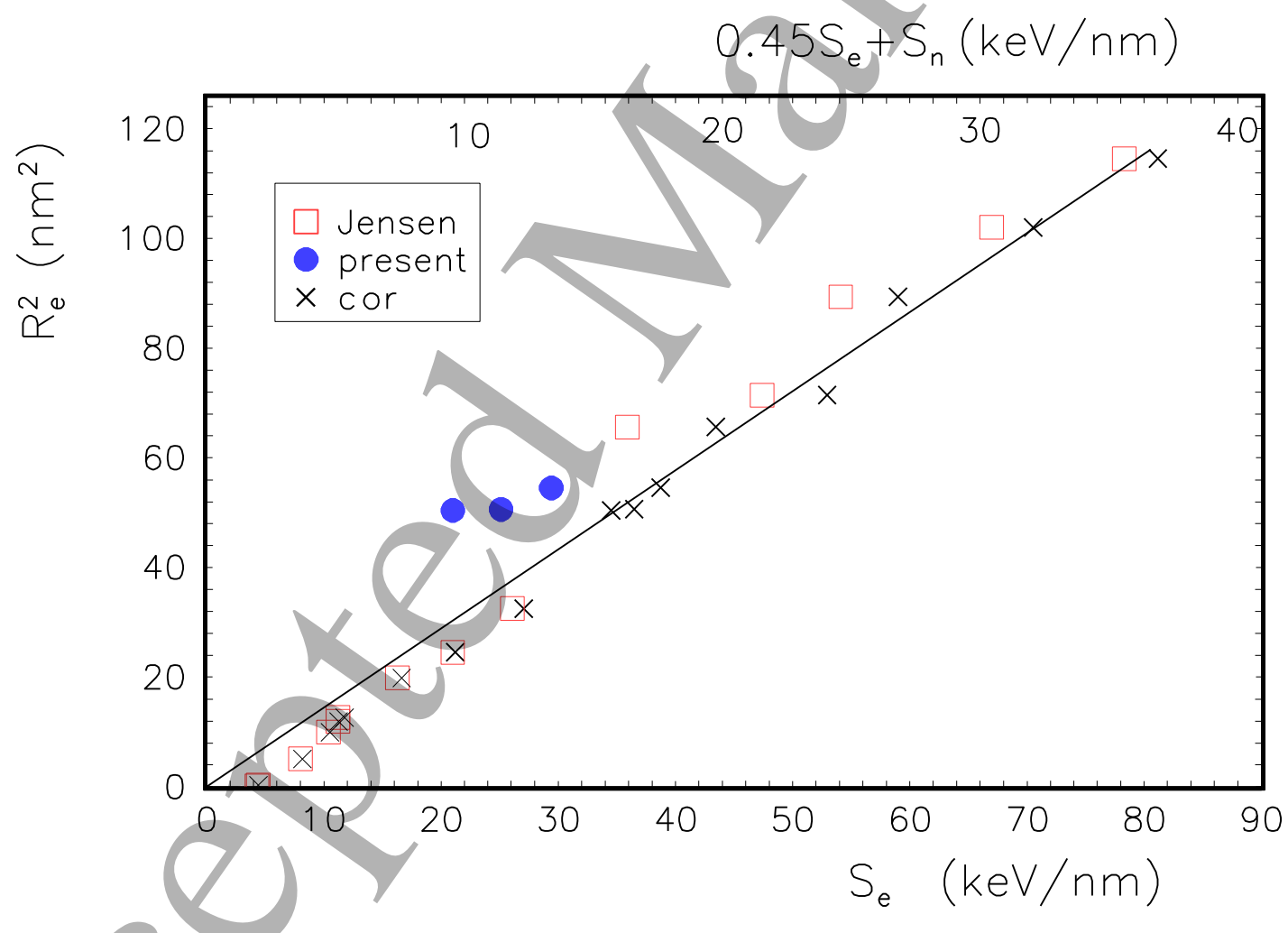

Figure 3. Variation of track radii $\mathrm{R}_{\mathrm{e}}$ with the electronic stopping power $\mathrm{S}_{\mathrm{e}}$ in $\mathrm{YIG}$ samples irradiated by Cn cluster beams of various energy. The experimental data were corrected taking into account the contribution of the nuclear stopping power $S_{n}(x)$; see upper scale. 
By applying the previous empirical relation it is assumed that the simultaneous effect of $S_{n}$ and $\mathrm{S}_{\mathrm{e}}$ can be described in the form of

$$
\mathrm{R}_{\mathrm{e}}^{2}=\mathrm{k}\left(\alpha \mathrm{S}_{\mathrm{e}}+\beta \mathrm{S}_{\mathrm{n}}\right)
$$

where $\mathrm{k}, \alpha$ and $\beta$ are constants whose values we intend to estimate from the analysis of experimental data. Eq.(1) can be transformed in the form

$$
\mathrm{R}_{\mathrm{e}}^{2} / \mathrm{S}_{\mathrm{e}}=\mathrm{k}\left(\alpha+\beta \frac{\mathrm{S}_{\mathrm{n}}}{\mathrm{S}_{\mathrm{e}}}\right)
$$

that provides an opportunity for a simple analysis. In Fig.4, track data of YIG are plotted according to Eq.(2). The results are $k \beta=3.2$ and $k \alpha=1.44$ leading to $\alpha / \beta=0.45$ (the lowest point was not used in the fit). This simple analysis led to an important result. Actually, when track formation processes are considered the energy deposition by elastic interaction is about twice as efficient than the electronic one. The deposited electronic energy is transformed to lattice energy by a rather complex way and it is possible that some processes do not contribute to the formation of tracks. On the other hand, the deposition of the elastic energy is rather localized for the low energy $\mathrm{Cn}$ ions. Therefore, it is reasonable 


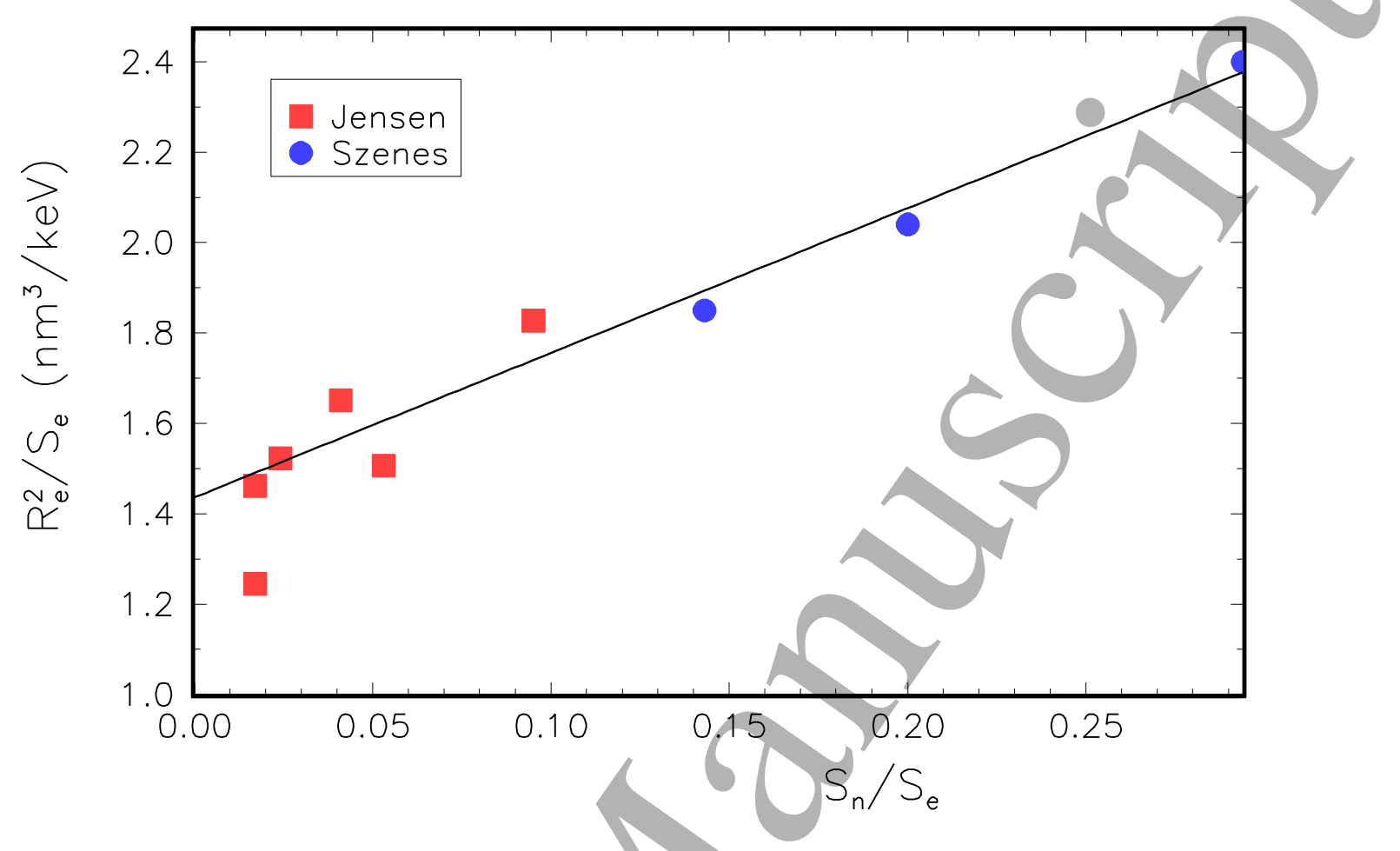

Figure 4. Analysis of track data by using Eq.(2); $R_{e}-$ is the track radius, $S_{n}$ and $S_{e}$ are the nuclear and the electronic stopping power.

to assume that $\beta \approx 1$ for our experiment and accordingly $\alpha \approx 0.45$.

The energy deposition processes must not be special in the range of 20-35 keV/nm. Therefore, it is assumed that our result is valid beyond this range as well even when $\mathrm{S}_{\mathrm{e}} \gg>\mathrm{S}_{\mathrm{n}}$. On the other hand, after studying the published results, we did not find marked difference in the behavior of YIG and other track forming insulators. This is an indication that $\alpha<1$ may be valid for other materials as well, though this still must be confirmed by direct measurements on other solids.

It wàs the basis of our analysis that the active fraction of the energy deposition leading to track formation is the linear combination of $\mathrm{S}_{\mathrm{e}}$ and $\mathrm{S}_{\mathrm{n}}$ and found that the correct form could be approximated by the expression $0.45 \mathrm{~S}_{\mathrm{e}}+\mathrm{S}_{\mathrm{n}}$. It is evident that the elastic contribution is rather high in some cases and 
the position of the track data may change rather considerably in the figure when $S_{e}>>S_{n}$ is not valid. In Fig.3, the track evolution curve becomes smoother, the deviations are reduced considerably and the curve is more coherent after introducing the correction term. In all irradiations resulting in $R_{\mathrm{e}}^{2}<30 \mathrm{~nm}^{2}$ C10, C5 and C2 cluster ions were applied with relatively high energies in Fig.3 [5]. Therefore, $S_{n} / S_{e}<<1$ was valid and the correction did not change the track positions in the figure. This also confirms the validity of our approach. We note that the track data for YIG follow nicely the $\mathrm{R}_{\mathrm{e}}{ }^{2}=\mathrm{kS}$ e line after the correction in Fig.3.

The value of $\alpha$ was estimated based on experiments on YIG. The method was rather sensitive to the accuracy of the track measurements and it was an advantage that we could use an internal calibration in YIG in the analysis of TEM pictures. It is shown that when track formation is the result of the simultaneous action of $S_{e}$ and $S_{n}$ then $\alpha S_{e}+S_{n}$ is a good approximation for Cn projectiles. In the case of clusters of heavier than carbon atoms the value of $\beta$ can be reduced due to the high energy collision cascades leading to a considerably broader energy distribution. It is possible to estimate the value of $\beta$ by applying the SRIM code [4]. This would require the knowledge of the mechanism of track formation as the size of the radial range around the trajectory ought to be known where $S_{n}$ may modify the track size. However, we just concentrate on obtaining independent experimental information that may reveal some details of the controlling mechanism. Thus we do not intend to apply any model in this paper.

Later in this paper we shall show other methods as well for the estimation of $\alpha$. Nevertheless, we can conclude at this stage that it is without doubts, that only a fraction of $S_{\mathrm{e}}$ is efficient for track formation. This is important, because this is not widely known though the simultaneous formation of several types of defects besides amorphous tracks have been described in a number of publications. However, it was not concluded in any case that these processes reduce the energy available for amorphous track formation. Moreover, there are theoretical calculations showing that excited elastic waves can carry off alone up to $50 \%$ of the initially deposited energy of a spherical spike [10] or up to $30 \%$ for a cylindrical spike [11]. Nevertheless, these and similar results have not been applied in various theories of track formation. We note that the above effects are in agreement with our present results. 


\subsection{Velocity effect}

It is a well-known experimental fact that low velocity ions are more efficient for track formation. This is the so-called velocity-effect (VE) [13]. There is not a generally accepted energy range for low velocity (LO) and high velocity (HI) ions. According to our experience the appropriate ranges are $\mathrm{E}<2$ $\mathrm{MeV} /$ nucleon and $\mathrm{E}>8 \mathrm{MeV} /$ nucleon for $\mathrm{LO}$ and $\mathrm{HI}$ ions, respectively. In these ranges, $\mathrm{VE}$ is approximately uniform within experimental error. Up until now, the track formation in the transition range $2<\mathrm{E}<8 \mathrm{MeV} /$ nucleon has not been studied in details.

The active fraction of the deposited energy $\alpha$ may vary with E. Unfortunately, the previous analysis cannot be repeated for experiments with swift heavy ions when $\mathrm{E}>8 \mathrm{MeV} /$ nucleon, as $\mathrm{S}_{\mathrm{e}}>>\mathrm{S}_{\mathrm{n}}$ in this range. We note that VE may vary with $S_{\mathrm{e}}$ even at $\mathrm{E} \approx$ constant in some complex structures [14] but those experiments are not included in this study. 


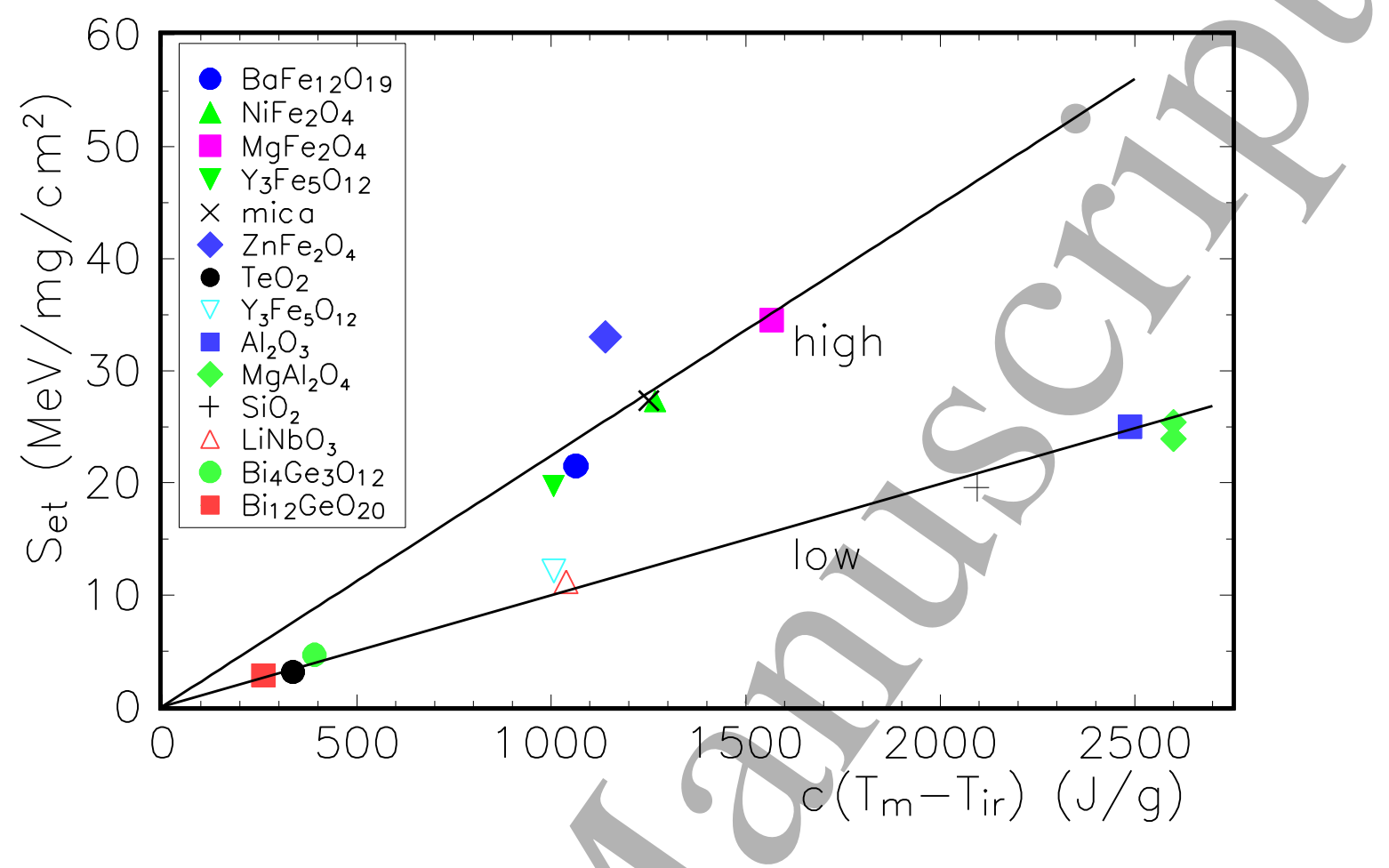

Fig.5. Variation of the threshold electronic stopping power $S_{\text {et }}$ in various insulators for low $(E<2$ $\mathrm{MeV} /$ nucleon) and high ( $\mathrm{E}>8 \mathrm{MeV} /$ nucleon) velocity ions; $\mathrm{c}, \mathrm{T}_{\mathrm{m}}$ and $\mathrm{T}_{\text {ir }}$ denote the specific heat, melting and irradiation temperatures, respectively (see Ref.[15] for details).

It is mentioned in the Introduction that we expect useful results from the comparison of the experiments performed on different insulators. One of these possibilities is the study shown in Fig.5 where threshold values of track formation $S_{e t}$ are shown in different insulators versus $c\left(T_{m}-T_{i r}\right)$ where $c$ is the specific heat, $\mathrm{T}_{\mathrm{m}}$ and $\mathrm{T}_{\mathrm{ir}}$ are the melting and the irradiation temperatures, respectively. The specific heat is estimated by applying the Neumann-Kopp rule.

It is highly important that the figure demonstrates the uniform behavior of insulators both at high and low ion velocities. This is an essential feature of track formation. In the previous section it was found that $\alpha \approx 0.45$ for YIG. The straight lines in Fig.5 demonstrate the similarity of the behavior of YIG 
and other insulators in both energy ranges. This is also supported by experiments on $\mathrm{Gd}_{2} \mathrm{Ti}_{2} \mathrm{O}_{7}$, $\mathrm{Gd}_{2} \mathrm{Zr}_{2} \mathrm{O}_{7}, \mathrm{Gd}_{2} \mathrm{TiZrO}_{7}$ where there is no drop on the $\mathrm{R}_{\mathrm{e}}-\mathrm{S}_{\mathrm{e}}$ track evolution curves when monoatomic and cluster ions are equally used in experiments [8]. One of the consequences of the plot in Fig.5 is that $\alpha<1$ is expected for other insulators as well.

Typically, $\mathrm{S}_{\mathrm{et}}$ is considerably lower for LO irradiations compared to experiments with $\mathrm{HI}$ ions. This is the consequence of VE. A relationship between $S_{\text {et }}$ and $T_{m}$ shown in the figure is not unexpected as the amorphous-crystalline phase transition takes place at this temperature in normal conditions. However, it is a very important information that $S_{\text {et }}$ is a simple function of $T_{m}$. The result is a clear demonstration of the thermal origin of track formation. It would be difficult to explain the role of $T_{m}$ on another basis.

In previous sections we found that only a fraction $\eta=\alpha S_{\text {et }}$ is directly related to the process of track formation. It is a reasonable assumption that $\alpha$ may be even lower in the HI range due to VE and the lower value of $\alpha$ is compensated by a higher $S_{\text {et. }}$ Thus the value of $\alpha$ in the HI range $\alpha_{\mathrm{HI}}$ can be simply estimated if the corresponding values of $S_{\text {et }}$ are known. We can use the slopes of the two lines in Fig. 5 for this purpose leading to $\alpha_{\mathrm{LO}} / \alpha_{\mathrm{HI}}=2.27$ [15]. Other experiments also support this explanations (see later).

The importance of this experiment is due to the fact that $\alpha=1$ is assumed in nearly all present theoretical approaches when track formation is considered. Therefore our experiment may be followed by similar ones on other insûlators.

\subsection{Relation between track sizes induced in different insulators}

Further basic information can be drawn from the plot in Fig.6 where again a relationship is presented between track formation in various insulators [16]. In this figure, track radii $\mathrm{R}_{\mathrm{e}}$ were induced by $\mathrm{HI}$ projectiles and $\left\langle\mathrm{s}_{\mathrm{e}}\right\rangle=\mathrm{A}=$ constant throughout the figure where $\left\langle\mathrm{s}_{\mathrm{e}}\right\rangle=\mathrm{S}_{\mathrm{e}} / \mathrm{N}$ 


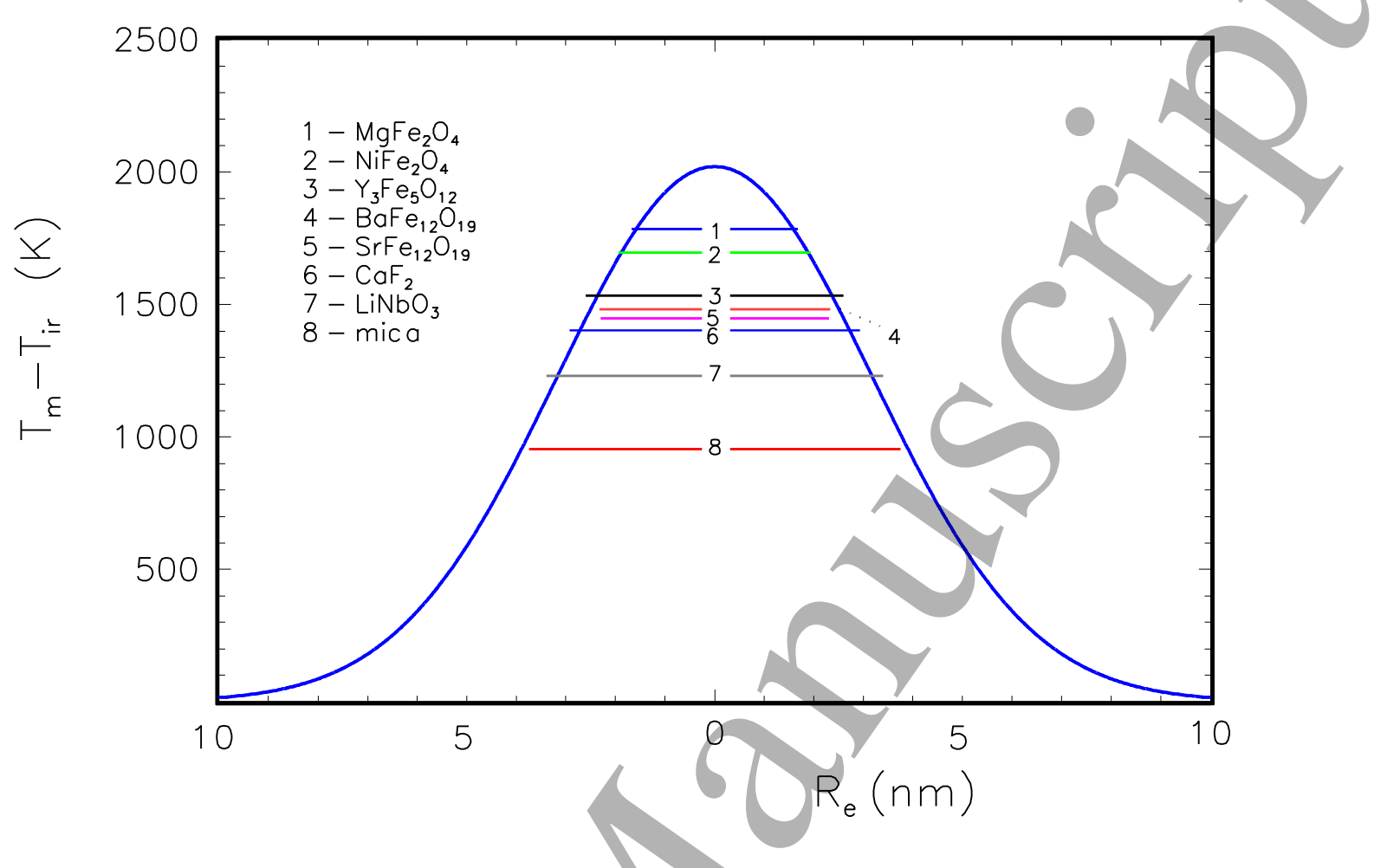

Figure 6. Variation of the track radii $\mathrm{R}_{\mathrm{e}}$ with $\mathrm{T}_{\mathrm{m}}-\mathrm{T}_{\mathrm{ir}}$ where $\mathrm{T}_{\mathrm{m}}$ and $\mathrm{T}_{\mathrm{ir}}$ denote the melting and irradiation temperatures, respectively; $\left\langle\mathrm{s}_{\mathrm{e}} / 3 \mathrm{k}\right\rangle=7.5 \times 10^{5} \mathrm{~nm}^{2} \mathrm{~K}$ for all data with $\left\langle\mathrm{s}_{\mathrm{e}}\right\rangle=\mathrm{S} / \mathrm{N}$, where $\mathrm{S}_{\mathrm{e}}$ and $\mathrm{N}$ are the electronic stopping power and the atomic density, respectively; for all data $\mathrm{E}>8 \mathrm{MeV} /$ nucleon. The enveloping curve given by $\Theta(r)=T_{p H} \exp \left\{-r^{2} /\left(w_{H}\right)^{2}\right\}$ is a fit with $\mathrm{w}_{\mathrm{H}}=4.5 \pm 0.27 \mathrm{~nm}$ and $\mathrm{T}_{\mathrm{pH}}=2020 \pm 100$ $\mathrm{K}$, for details see [16].

and $\mathrm{N}$ is the number density of atoms. The only exception was $\mathrm{CaF}_{2}$ where $\mathrm{Cn}$ cluster irradiations were applied in the LO range, nevertheless, the results were coherent with the track data of other insulators induced in the $\mathrm{HI}$ range. $\mathrm{CaF}_{2}$ is included into this figure just because it is an important exception (see later). In these experiments, the irradiations were performed in different solids using different ions with different energies, with different values of $S_{\mathrm{e}}$. Nevertheless, there is a simple quantitative relationship 
between track radii induced in nominally independent experiments. This must be related to a basic feature of the underlying mechanism.

In Fig.6, the variation of track radii $R_{e}$ can be described by a simple Gaussian curve $\Theta(\mathrm{r})=\mathrm{T}_{\mathrm{pH}} \exp \left\{-\mathrm{r} / \mathrm{w}_{\mathrm{H}}\right\}^{2}$, where $\mathrm{w}_{\mathrm{H}}=4.5 \pm 0.27 \mathrm{~nm}$ and the peak temperature $\mathrm{T}_{\mathrm{pH}}=2020 \pm 100 \mathrm{~K}$. According to the figure $2 R_{e}$ is equal to the width of the $\Theta(r)$ function at $\Theta=T_{m}-T_{\text {ir }}$ and this procedure provides the appropriate track radii for various insulators within experimental error. Similarly to the previous figure the variable is $T_{m}$ in this plot proving the thermal origin of track formation and its relationship with the ion-induced temperature. This is a very important basic result that has not received any attention from theoreticians in the last ten years up until now. A further important information given by this figure is that $<\mathrm{s}_{\mathrm{e}}>$ and do not $\mathrm{S}_{\mathrm{e}}$ is the correct parameter when track formation is studied in experiments on several insulators. The existence of the $\Theta(r)$ relation is rather convincing in this respect.

The plot in Fig.6 is highly valuable as it demonstrates that when the temperature sensitivity is examined in a particular solid, track formation is related exclusively to $\mathrm{T}_{\mathrm{m}}$. There is no indication for any insulator in Fig.6, that any thermal parameter which is specific to a given solid apart $\mathrm{T}_{\mathrm{m}}$ would affect the track size. Evidently, this feature is valid in other presentations of the track data, as well. Therefore, if an $\mathrm{R}_{\mathrm{e}}{ }^{2}-\mathrm{S}_{\mathrm{e}}$ track evolution curve is studied separately one cannot expect obtaining information on any other parameter of the ion induced temperature distribution $T(r, t)$ as $R_{e}$ does not depend on it. Consequently, there seems to be no requirement from the experimental side on the shape, the peak temperature or the thermal energy of $\mathrm{T}(\mathrm{r}, \mathrm{t})$ or on the contribution of the heat of fusion when tracks are studied only in a particular solid. The only information we have is that the width of $\Theta(r)$ is equal to $2 R_{e}$ at $\mathrm{T}_{\mathrm{m}}-\mathrm{T}_{\text {ir }}$ and this is an indication that the maximum width of $\mathrm{T}(\mathrm{r}, \mathrm{t})$ is equal to the track diameter when the local temperature $\mathrm{T}=\mathrm{T}_{\mathrm{m}}$

There is a serious problem hidden in these observations. The induced temperature does not depend exclusively on the properties of the particular solid. In reality, further restriction must exist for $\mathrm{T}(\mathrm{r}, \mathrm{t})$ as its parameters cannot vary freely from the relation $\Theta(\mathrm{r})$. This is valid for a number of insulators, as well, otherwise, the existence of $\Theta(r)$ would be impossible. Thus the temperatures induced in different 
insulators are indirectly related due to their dependence on $\Theta(r)$. And this is the key to the solution of the problem: the plot in Fig. 6 demonstrates the existence of a relationship between $\Theta(r)$ and the induced temperatures in different solids. The experiments provide information on the parameters of $T(r, t)$ in a broad range of temperatures, however, it can be revealed only by a complex method involving studies on different solids and their simultaneous analysis like in Fig.6.

The effect of the heat of fusion $L$ on the track size is a characteristic situation from this aspect. It is evident from the plot in Fig.6 that track radii $R_{\mathrm{e}}$ depend only on a single variable $\mathbf{T}_{\mathrm{m}}$. Thus the melting temperature $\mathrm{T}_{\mathrm{m}}$ is the unique MP that affects the track size. If this were not correct, considerable deviation from the smooth curve would appear in Fig.6 as MPs, including the heat of fusion L, usually, are not proportional to $\mathrm{T}_{\mathrm{m}}-\mathrm{T}_{\mathrm{ir}}$. However, there is no scatter exceeding the usual experimental error for the insulators in the figure. The same considerations are valid for other MPs, as well.

Compared to the heat $\mathrm{Q}_{\mathrm{m}}$ required for raising the temperature from room temperature to $\mathrm{T}_{\mathrm{m}}, \mathrm{L}$ may have a value up to $L / \mathrm{Q}_{\mathrm{m}} \approx 0.5$ and even higher [8]. Thus its value might affect the induced temperature considerably. However, different calculations of the track evolution curve e.g. in YIG $[12,17]$, provide equally good agreement with the experiments when $\mathrm{L}$ is taken into account [12] or ignored [17]. This is quite unexpected considering the high values of L. Moreover, it will be shown further in this paper, that the width, the peak temperature and the shape of $T(r, t)$ are strictly defined by the relationship between experimental data in various insulators in Fig.6. Nevertheless, it has not any effect on the accuracy of the calculations of $R_{e}$ when these requirements are ignored in a particular solid $[12,17]$. The reason of it has been clarified in the above considerations. In a theoretical analysis, only the maximum width of the calculated $\mathrm{T}(\mathrm{r}, \mathrm{t})$ must be correct at the melting temperature for obtaining the proper value of $\mathrm{R}_{\mathrm{e}}$ in a particular insulator and this can be achieved by a high number of different $\mathrm{T}(\mathrm{r}, \mathrm{t})$ functions. The application of all these $\mathrm{T}(\mathrm{r}, \mathrm{t})$ functions may lead to satisfactory agreement with track data when using suitable fitting parameters. However, it is only the track formation that has such a specific feature. The peak temperature and shape of $\mathrm{T}(\mathrm{r}, \mathrm{t})$ are, for example, indispensable information for the investigation of the electronic mixing or sputtering. 
Similarly to Fig.6 an analogous relationship was found for tracks induced by LO irradiations in various solids for $\left\langle\mathrm{s}_{\mathrm{e}}\right\rangle=\mathrm{B}=$ constant which also could be described by a Gaussian function with $\mathrm{w}_{\mathrm{L}}=4.45$ $\pm 0.18 \mathrm{~nm}[16]$. As a result of a proper choice of $\mathrm{B}$ the peak temperature $\mathrm{T}_{\mathrm{pL}}=2024 \pm 90 \mathrm{~K}$ was close to the value of $\mathrm{T}_{\mathrm{pH}}[16]$ with $\mathrm{B}<\mathrm{A}$ due to $\mathrm{VE}$. We emphasize here that $\mathrm{w}=4.5 \mathrm{~nm}$ is identical for various insulators, and it does not vary with the ion specific energy E. A detailed analysis showed that $\left\langle\mathrm{se}_{\mathrm{e}}\right\rangle$ also may vary in a broad range keeping $\mathrm{w}=4.5 \mathrm{~nm}$ [17]. Previously, we showed that $\mathrm{w}$ may be related to fundamental constants namely

$$
\mathrm{a}_{0}=\frac{\lambda_{\mathrm{e}}}{2 \pi \alpha} \approx \frac{\pi \alpha}{2} \mathrm{w}
$$

where $\mathrm{a}_{0}, \lambda_{\mathrm{e}}$ and $\alpha$ are the Bohr radius, the Compton wavelength of electrons and the fine structure constant, respectively. Eq.(3) provides $\mathrm{w}=4.62 \mathrm{~nm}$ in excellent agreement with the experiments showing a deviation within 3\% [16]. This may be considered as an indication that the existence of the $\Theta(r)$ relation is the consequence of basic interactions which have not been taken into account previously.

$T(r, t)$ is the ion-induced temperature increase and its value is $T_{m}-T_{\text {ir }}$ when the local temperature is $T_{m}$. The width of $T(r, t)$ varies with time; we take for the origin of the timescale $t=0$ the moment when the maximum width is attained at the melting temperature. In an ideal case, this maximum width is equal to the track diameter and in the same time it is equal to the width of the $\Theta(r)$ function at $\Theta=T_{m}-T_{\text {ir }}$ providing $\Theta\left(R_{e}\right)=T\left(R_{e}, 0\right)$ for the given solid. Moreover, this ought to be fulfilled simultaneously for $n$ solids with the same value of $\left\langle\mathrm{s}_{\mathrm{e}}\right\rangle$, having different thermal parameters and different $\mathrm{T}_{\mathrm{n}}(\mathrm{r}, 0)$ distributions since Fig. 6 and other similar figures are valid for a number of solids simultaneously. And this must be valid for various values of $\left\langle\mathrm{se}_{\mathrm{e}}\right\rangle=$ constant, as well. Thus we arrived to the conclusion that track radii in various insulators are given by the same universal-type function $\Theta(r)$.

Additionally, there is a further problem. $\mathrm{T}_{\mathrm{m}}$ can be considered as a preferred temperature only in that sense that it is a unique temperature where the width of $\mathrm{T}(\mathrm{r}, 0)$ can be measured indirectly using the appropriate track size. There is no such possibility for $\mathrm{T} \neq \mathrm{T}_{\mathrm{m}}$. Otherwise, all temperatures are equivalent for the energy deposition. Merely the fact that $\mathrm{T}_{\mathrm{m}}$ is the temperature of the phase transition 
is not a firm physical basis for justifying that the maximum widths of the $T_{n}(r, 0)$ distributions must be equal to a universal-type function $\Theta(r)$ at these temperatures for a number of solids. If the phase transition were responsible for this effect, then other parameters like heat of fusion, and various parameters of the amorphous phase would modify the $\Theta\left(R_{e}\right)=T\left(R_{e}, 0\right)$ equation. However, this is not the case.

Thus the relationship between $\mathrm{T}(\mathrm{r}, 0)$ and $\Theta(\mathrm{r})$ must be valid not only for a single temperature $\mathrm{T}_{\mathrm{m}}$ but for other temperatures as well. However, if the widths of $\mathrm{T}(\mathrm{r}, 0)$ and $\Theta(r)$ are equal in a solid at any temperature then they must be identical. This is essentially a mathematical condition deduced from Fig.6. The result is rather unexpected, but this is the only solution of the problems.

Thus $\Theta(r)$ provides reliable information on the shape and parameters of the ion-induced temperature, that is supported by experiments on numerous materials. It is evident from the above considerations that $\Theta(r)=T(r, 0)$ is an identical Gaussian distribution in a number of insulators as shown in Fig.6 and the Gaussian width $w$ do not vary with $\mathrm{E}$ or $\left\langle\mathrm{s}_{\mathrm{e}}\right\rangle$. In the present paper these are not assumptions of a model, but a conclusion drawn from well-known experimental facts. In the opposite case when $\Theta(r) \neq T(r, 0)$, the agreement of the experimental data with the Gaussian curve in Fig.6 would be the result of occasional coincidences that have an extremely low probability. We discussed this problem in Ref. [16] in details.

Continuing our considerations on $\Theta(r)$, we can also find information on the value of $\alpha$ in the HI range, as well. We have two sets of parameters for $\Theta(r)$ : one for LO and another for HI irradiations. It was found that the Gaussian width of $\mathrm{T}(\mathrm{r}, 0)=\Theta(\mathrm{r})$ does not vary with ion energy and $\mathrm{T}_{\mathrm{pL}} \approx \mathrm{T}_{\mathrm{pH}}$ in our case due to the appropriate yalues of $\mathrm{A}$ and $\mathrm{B}$. Thus the thermal energies are equal for any $\mathrm{T}_{\mathrm{n}}(\mathrm{r}, 0)$ distributions of $n$ solids and consequently $\alpha_{H I} B=\alpha_{L O} A$. By using the numerical value in [16] $\left(<\mathrm{s}_{\mathrm{e}} / 3 \mathrm{k}\right\rangle=3.42 \times 10^{5} \mathrm{~nm}^{2} \mathrm{~K}$ for LO irradiations $) \alpha_{\mathrm{HI}}=0.46 \alpha_{\mathrm{LO}}$. This is in good agreement with our previous estimate based on the variation of $\left\langle\mathrm{s}_{\mathrm{et}}\right\rangle$. Thus we arrived to a similar conclusion as previously when the plot in Fig.5 was used for the estimation of $\alpha_{\mathrm{HI}}$. This result is based on measurements on a 
considerable number of solids. The agreement between the two independent methods is very important as it confirms the reliability of our results with respect to $T(r, 0)=\Theta(r)$ and the values of $\alpha$.

A useful possibility is offered when the equation of the balance of energy is applied to $\Theta(r)$ as the source of the thermal energy is $\left.\alpha \mathrm{N}<\mathrm{s}_{\mathrm{e}}\right\rangle$. The dependence of the peak temperature $\mathrm{T}_{\mathrm{p}}$ on $\left\langle\mathrm{s}_{\mathrm{e}}\right\rangle$ can be obtained by this method and a complete analytical form of $\Theta(r)$ can be derived leading to

$$
\Theta(r)=\frac{\alpha<s_{e}>}{3 \pi k w^{2}} e^{-\left\{r^{2} / w^{2}\right\}}
$$

where $\mathrm{k}$ is the Boltzmann constant and the Neumann-Kopp law is used. An expression for $\left\langle\mathrm{s}_{\mathrm{et}}\right\rangle$ can be derived from Eq.(4) which is in good agreement with the data in the plot in Fig.5. A byproduct of Eq.(4) is that the value of $\alpha$ can be estimated from the peak temperatures $T_{p H}$ and $T_{p L}$ in Fig. 6 and in an analogous figure in Ref.[16]. The result is $\alpha_{\mathrm{LO}}=0.38$ and $\alpha_{\mathrm{HI}}=0.17$. This/value of $\alpha_{\mathrm{LO}}$ is slightly lower than our previous estimate using the plot on Fig.4. We consider the lower value more reliable as it is based on experiments on several materials and $\alpha_{\mathrm{LO}}=0.4$ is used in most publications.

The validity of the above result is rather simple to check. When $T_{m}$ and $R_{e}$ are known for an insulator in Fig. 6 and $T_{m}$ is also given for another one then $R_{e}$ can be easily calculated for it by using the expression for $\Theta$. When repeating this calculation for various values of $\left\langle\mathrm{s}_{\mathrm{e}}\right\rangle$ the complete $\mathrm{R}_{\mathrm{e}}\left(\left\langle\mathrm{s}_{\mathrm{e}}\right\rangle\right)$ track evolution curve can be obtained by this method. This has been done for several solids and good agreement between the experimental and calculated values was obtained even when track sizes in the HI range were estimated from track values in the LO range [18]. On one hand, this confirms our conclusions concerning the general features of $\Theta(r)$. On the other hand, it excludes the possibility that $\mathrm{R}_{\mathrm{e}}$ may depend on any other MPs besides $\mathrm{T}_{\mathrm{m}}$. We note that such calculations have been also done successfully for $\mathrm{Y}_{3} \mathrm{Fe}_{5} \mathrm{O}_{12}$ in Ref.[18] which is a basic material for experimental and theoretical studies often using about 10 various MPs.

A further possible step may be the application of the results for $\Theta=T_{m}-T_{i r}$ and $r=R_{e}$ leading to an analytical expression describing the $\mathrm{R}_{\mathrm{e}}=\mathrm{R}_{\mathrm{e}}\left(\left\langle\mathrm{s}_{\mathrm{e}}\right\rangle\right)$ track evolution curve. However, this is beyond the scope of this paper. 


\subsection{Final comments}

We mention, that the expression for $T(r, 0)=\Theta(r)$ has been successfully applied for the description of the ion-induced sputtering in $\mathrm{SiO}_{2}[19]$ and $\mathrm{UO}_{2}[20]$ in a broad range of $\left\langle\mathrm{s}_{\mathrm{e}}\right\rangle$ varying by an order of magnitude. In the case of track formation the key parameter is the maximum width at $T=T_{m}$, and the peak temperature of $\mathrm{T}(\mathrm{r}, \mathrm{t})$ is rather indifferent. However, the key parameter for electronic sputtering is rather the peak temperature. Our present results predict that the application of other calculated $\mathrm{T}(\mathrm{r}, \mathrm{t})$ distributions which seem to be successful in the analysis of track formation but are different from $\Theta(r)$ may lead to difficulties for sputtering while Eq.(4) is equally useful in sputtering and track studies $[19,20]$ in a broad range of $S_{e}$.

We have no doubts that the above conclusions are valid for those solids whose track data have been used in the analysis. Altogether, these are about 20 track forming insulators, which are about 1/3 of those solids in which irradiation induced tracks were found up until now if semiconductors, HTCSs and polymers are not included. However, this does not mean that the rest of the data are in contradiction with the above findings. Unfortunately, those data are not suitable for our analysis as $\mathrm{S}_{\mathrm{e}}$ or $\mathrm{E}$ or $\mathrm{N}$ or $\mathrm{T}_{\mathrm{m}}$ are not well defined in the experiments or the measurements are performed in the range $2 \mathrm{MeV} /$ nucleon $<\mathrm{E}<8 \mathrm{MeV} /$ nucleon where the $\alpha(\mathrm{E})$ function is not known at present with sufficient accuracy. According to our best knowledge, there is not any reliable systematic experimental study contradicting to our above conclusions.

The main question is what is the significance of the uniform behavior of the above 20 insulators, in general? We note that it is a rather unique phenomenon when complex processes proceed identically in various solids without any effect of the individual materials properties. This is a new effect, no similar behavior has been described or predicted by any microscopic model or theoretical simulation. This is remarkable even when there would be no more such solids than those 20. However, as these solids were chosen essentially randomly for experiments in the original publications it is a reasonable assumption that a rather high number of insulators may exist with such properties. When extending the systematic 
investigations to other materials, it would be important to find insulators where $\Theta(r)$ is not formed since this may assist revealing the origin of the actual mechanism of the localized energy deposition.

While the amorphous tracks are apparently stable in the above solids, there are also examples when some transformations may occur within the tracks. There are materials where partial recrystallization is observed $\left(\mathrm{Al}_{2} \mathrm{O}_{3}, \mathrm{CeO}_{2}\right.$ [14] $)$ and tracks with complex structure are formed in others. Thus tracks consisting of an amorphous core and a disordered defect-fluorite shell are reported recently in pyrochlors where the fraction of these components varies with $\left\langle\mathrm{s}_{\mathrm{e}}\right\rangle$ [21]. Some other deviations from the general behavior are also reported. For example, there are evidences that VE is missing in some ionic crystals. An evidence is seen in Fig.6 where $\mathrm{LO}$ track radius for $\mathrm{CaF}_{2}$ fits quite well in the Gaussian curve for the $\mathrm{HI}$ range. VE is also missing in the overwhelming majority of other experimental data in this solid including TEM, Rutherford backscattering and X-ray diffraction data [22,23]. Recent track data [24] are also in agreement within experimental error with the one used in Fig.6. However, there are opposite opinions as well based on a few $\mathrm{HI}$ tracks in $\mathrm{CaF}_{2}$ [23] while no explanation is given why is VE missing in most of the author's data. Further experiments should solve this discrepancy as there are also other results indicating the missing of VE in ionic crystals [25]. Besides the ionic crystals, there may be other types of insulators with missing VE as well. Then $\Theta(r)$ is identical to $T(r, 0)$ for them in the $\mathrm{HI}$ and $\mathrm{LO}$ ranges as well as $\alpha=\alpha_{H I}$ in the full range of ion velocities in this case.

When analyzing directly the raw experimental data we did not use any assumptions. Therefore, the results show real quantitative relations which exist between the experimental data. These quantitative relations must be valid for the results of the theoretical calculations as well. A theoretical model that describes correctly the physical processes must be inherently coherent with these findings.

It is interesting that the heat of fusion $\mathrm{L}$ seems to be indifferent for track formation though the crystalline-amorphous phase transition is a basic process. The absence of $\mathrm{L}$ may be the consequence of a more general problem: equilibrium values of thermal parameters are not valid in the conditions of extremely intensive and fast electronic processes. Superheating is not the solution of the problem as the melting temperatures $\mathrm{T}_{\mathrm{m}}$ represent the MPs in Figs.5,6. This situation deserves a thorough theoretical analysis. 


\section{Conclusions}

Prethinned TEM samples of YIG were irradiated by C60 beams of 3.5, 5 and $7 \mathrm{MeV}$ energy and the diameters of the induced amorphous tracks were measured. Previous experimental data obtained by irradiations with $\mathrm{Cn}$ cluster beams were analyzed together with our new results. Quantitative relationships are derived without the applications of any model. By using the results of TEM measurements it is shown that only an $\alpha$ fraction of $S_{\mathrm{e}}$ takes part in the processes of track formation. A comparison with the experiments with swift heavy ions showed for insulators that $\alpha$ varies with $\mathrm{E}$ from $\alpha_{\mathrm{LO}} \approx 0.4$ (E<2 MeV/nucleon) to $\alpha_{\mathrm{HI}} \approx 0.17$ (E>8 MeV/nucleon). This provides an explanation of the velocity effect. In the case of a simultaneous effect the expression $0.4 S_{e}+\beta S_{n}$ is responsible for track formation where $\beta \approx 1$ for C60 cluster ions. A universal-type $\Theta(r)$ rélationship is found between independent experimental $\mathrm{R}_{\mathrm{e}}, \mathrm{T}_{\mathrm{m}}$ data pairs measured in various insulators for $\left\langle\mathrm{s}_{\mathrm{e}}\right\rangle=$ constant; $\Theta(r)=\alpha<s_{e}>/ 3 \pi k w^{2} \exp \left\{-r^{2} / w^{2}\right\}$ with $w=4.5 \mathrm{~nm}$ providing $r=R_{e}$ for $\Theta(r)=T_{m}-T_{i r}$ for a number of trackforming insulators.

It is concluded that the ion-induced $\mathrm{T}(\mathrm{r}, 0)$ temperature distributions are identical in different insulators: $\mathrm{T}(\mathrm{r}, 0)=\Theta(r)$ for $\alpha<\mathrm{s}_{\mathrm{e}}>=$ constant. $\mathrm{T}_{\mathrm{m}}$ is the only MP including the heat of fusion $\mathrm{L}$ that controls the track size indicating the thermal origin of track formation. The width and shape of $T(r, 0)$ do not change with $\mathrm{E}$ and $\mathrm{S}_{\mathrm{e}}$ and $\left\langle\mathrm{S}_{\mathrm{e}}\right\rangle$ rather than $\mathrm{S}_{\mathrm{e}}$ is the right parameter when tracks are studied simultaneously in several insulators. As the above conclusions are derived without any assumptions directly from the raw experimental data of about 20 insulators they must reappear in a correct model of irradiation effects, as well.

\section{Acknowledgements}

The authors are grateful to Dr. M. Döbeli (ETH, Zürich) for careful irradiation of the samples by C60 beams. 
References

[1] Ion Beam Science: Solved and Unsolved Problems 2006 ed Sigmund P Matematisk-fysiske Meddelelser 52 Copenhagen

[2] Dufour C and Toulemonde M 2016 Models for the Description of Track Formation In Ion Beam Modification of Solids ed W Wesch and E Wendler Springer Series in Surface Sciences vol 61 pp 63104 Springer Cham

[3] Introduction by A Einstein to Johannes Kepler: life and letters 1951 by Baumgardt C Philosophical Library NY

[4] Ziegler J F www.SRIM.org

[5] Jensen J Dunlop A Della-Negra S Toulemonde M 1998 Nucl. Instrum. and Meth. B 146412

[6] Szenes G 2002 Nucl. Instrum. and Meth. B 191186

[7] Jozwik-Biala I Jagielski J Thomé L Arey B Kovarik L Sattonnay G Debelle A Monnet I 2012 Nucl. Instrum. and Meth. B 286258

[8] Zhang J M Toulemonde M Lang M Costantini J M Della-Negra S Ewing RC 2015 J Mater Res 30 2456

[9] Meftah A Costantini J M Khalfaoui N Boudjadar S Stoquert J P Studer F and Toulemonde M 2005 Nucl. Instrum. and Meth. B 237563

[10] Martynenko Yu V and Umansky M V 1994 Radiat. Eff. Def. Sol. 13231

[11] Jakas M and Bringa EM 2000 Phys. Rev. B 62824 
[12] Toulemonde M Assmann W Dufour C Meftah A Studer F Trautmann C 2006 Matematisk-fysiske Meddelelser $\mathbf{5 2} 263$

[13] Meftah A Brisard F Costantini JM Hage-Ali M Stoquert J P and Toulemonde M 1993 Phys. Rev. B 48920

[14] Szenes G and Pécz B 2016 Nucl. Instrum. and Meth. B 389-390 17

[15] Szenes G 2007 Rad. Eff. Defects Sol. 162557

[16] Szenes G 2012 Nucl. Instrum. and Meth. B 28088

[17] Szenes G 2005 J. Nucl. Mater. 33681

[18] Szenes G 2013 Nucl. Instrum. and Meth. B 312118

[19] Szenes G 2005 Nucl. Instrum. and Meth. B 23370

[20] Szenes G 2016 J. Nucl. Mater. 48228

[21] Lang M et al 2014 Nucl. Instrum. and Meth. B 336102

[22] Szenes G 2013 Phys. Rev. B 87056101

[23] Toulemonde M et al 2012 Phys. Rev. B 85054112

[24] Ishikawa N Taguchi T and Okubo N 2017 Nanotechnology 28445708

[25] Szenes G 2000 Phys. Rev. B 6114267 


\section{Captions}

Figure 1. Bright field TEM picture of a YIG sample irradiated by $\mathrm{C} 60$ ions of $5 \mathrm{MeV}$ energy. Insert shows magnified image of the YIG crystal in $\{111\}$ orientation with a circular amorphous track.

Figure 2. HREM picture of two closely positioned track in a YIG sample irradiated by C60 ions of 3.5 $\mathrm{MeV}$ energy.

Figure 3. Variation of track radii $R_{e}$ with the electronic stopping power $S_{e}$ in $Y I G$ samples irradiated by $\mathrm{C}_{\mathrm{n}}$ cluster beams of various energy. The experimental data were corrected taking into account the contribution of the nuclear stopping power $S_{n}(x)$; see upper scale.

Figure 4. Analysis of track data by using Eq.(2); $R_{e}-$ is the track radius, $S_{n}$ and $S_{e}$ are the nuclear and the electronic stopping power.

Figure 5 Variation of the threshold electronic stopping power $S_{\text {et }}$ in various insulators for low $(E<2$ $\mathrm{MeV} /$ nucleon) and high ( $\mathrm{E}>8 \mathrm{MeV} /$ nucleon) velocity ions; $\mathrm{c}, \mathrm{T}_{\mathrm{m}}$ and $\mathrm{T}_{\mathrm{ir}}$ denote the specific heat, melting and irradiation temperatures, respectively (see Ref.[15] for details).

Figure 6. Variation of the track radii $\mathrm{R}_{\mathrm{e}}$ with $\mathrm{T}_{\mathrm{m}}-\mathrm{T}_{\mathrm{ir}}$ where $\mathrm{T}_{\mathrm{m}}$ and $\mathrm{T}_{\mathrm{ir}}$ denote the melting and irradiation temperatures, respectively; $\left\langle\mathrm{s}_{\mathrm{e}} / 3 \mathrm{k}\right\rangle=7.5 \times 10^{5} \mathrm{~nm}^{2} \mathrm{~K}$ for all data with $\left\langle\mathrm{s}_{\mathrm{e}}\right\rangle=\mathrm{S} / \mathrm{N}$, where $\mathrm{S}_{\mathrm{e}}$ and $\mathrm{N}$ are the electronic stopping power and the atomic density, respectively; for all data $\mathrm{E}>8 \mathrm{MeV} /$ nucleon. The enveloping curve given by $\Theta(r)=T_{p H} \exp \left\{-r^{2} / w^{2}\right\}$ is a fit with $\mathrm{w}_{\mathrm{H}}=4.5 \pm 0.27 \mathrm{~nm}$ and $\mathrm{T}_{\mathrm{pH}}=2020 \pm 100$ $\mathrm{K}$, for details see [16]. 


\section{Table}

Table 1. Irradiation parameters of prethinned TEM samples; $\mathrm{R}_{\mathrm{e}}$ - track radius. 